\title{
A Novel and Facile Method to Synthesize Self-Assembled BiOCl Core-shell Microspheres Composed of Nanoplates
}

\author{
Guang LU *, Zishuai LUN, Hongyu LIANG, Fei WANG, Hui WANG \\ College of Chemistry, Chemical Engineering and Environmental Engineering, Liaoning Shihua University, Fushun, 113001, \\ China \\ crossref http://dx.doi.org/10.5755/j01.ms.25.1.19374
}

Received 27 October 2017; accepted 13 January 2018

\begin{abstract}
The self-assembled $\mathrm{BiOCl}$ core-shell microspheres composed of nanoplates have been successfully prepared using solvothermal method using ethylene glycol and polyvinylpyrrolidone (PVP) as solvents. XRD, SEM, EDX, XPS and DRS characterizations have been performed to study the crystal structure, morphology, composition and optical property of the as-prepared $\mathrm{BiOCl}$ materials. The XRD results indicate that the obtained $\mathrm{BiOCl}$ materials are high-purity and single-phase. The SEM results show that the as-prepared $\mathrm{BiOCl}$ powders are core-shell microspheres with diameters about $1 \mu \mathrm{m}$, and the core and shell of these microspheres are composed of many longitudinal grown nanoplates. The EDX and XPS results reveal that the atomic ratio of $\mathrm{Bi} / \mathrm{O} / \mathrm{Cl}$ in the sample is approximately equal to $1: 1: 1$. Moreover, the DRS spectra reveal that the band gap of the resulting sample is estimated to be about $2.92 \mathrm{eV}$. The results of photocatalytic activity show that the as-prepared $\mathrm{BiOCl}$ core-shell microspheres exhibit more excellent activity than P25 (Degussa, commercial $\mathrm{TiO}_{2}$ powder manufactured by flame hydrolysis) under visible light irradiation.

Keywords: $\mathrm{BiOCl}$, core-shell microspheres, solvothermal method, crystal structure, XPS.
\end{abstract}

\section{INTRODUCTION}

Bismuth oxychloride (BiOCl) has been attracted many attentions owing to its potential photocatalysis application $[1,2]$, besides being applied in ionic conduction, ferroelectric materials and pigments $[3,4]$. Therefore, various synthesis methods have been developed successfully to prepare $\mathrm{BiOCl}$ samples, such as hydrolysis process [5], reverse micro-emulsion synthesis [6] and solvothermal process [7]. The obtained $\mathrm{BiOCl}$ samples present different morphologies including nano-scale particle, nanosheet and micro-scale morphology assembled with nanosheet. For instance, Deng Z.-T. et al. have firstly reported that two-dimensional (2D) single-crystalline bismuth oxyhalides ( $\mathrm{BiOX}, \mathrm{X}=\mathrm{Cl}, \mathrm{Br})$ micro- and nanostructures, such as nanoplates, nanosheets, and microsheets, were synthesized in a large scale by a simple wetchemistry approach of hydrogen peroxide $\left(\mathrm{H}_{2} \mathrm{O}_{2}\right)$ direct oxidation of bulk metal bismuth (Bi) particles in a mixed solution followed [8]. Ding L.-Y. et al. have reported uniform $\mathrm{BiOCl}$ hierarchical microspheres assembled by nanosheets with tunable thickness were synthesizedvia a simple solvothermal route using acetic acid and methanol as solvents [9]. Hao H.-Y. et al. obtained $\mathrm{BiOCl}$ microspheres and microflowers by a simple solvothermal method using bismuth nitrate and sodium chloride as solvent, respectively [10]. Wu Y.-P. et al. obtained $\mathrm{BiOCl}$ microrings composed of nanoplatelets via a simple hydrothermal method using an oleic acid contained solvent as a reaction medium [11]. However, to the best of our knowledge, there has not been a study on the preparation of $\mathrm{BiOCl} 3 \mathrm{D}$ microspheres with core-shell structure so far.
In this study, $\mathrm{BiOCl}$ core-shell microspheres were synthesized by solvothermal method using ethylene glycol and PVP as solvents. In addition, the phase structure, morphology and composition of the resulting $\mathrm{BiOCl}$ sample were investigated in detail.

\section{EXPERIMENT}

\subsection{Preparations of $\mathrm{BiOCl}$ microspheres}

$\mathrm{BiOCl}$ microspheres with core-shell structure were prepared via solvothermal method. $0.2 \mathrm{~g} \mathrm{Bi}\left(\mathrm{NO}_{3}\right)_{3} \cdot 5 \mathrm{H}_{2} \mathrm{O}$ was added intomixture solution of ethylene glycol and polyvinylpyrrolidone (PVP). Then, the hydrochloric acid containing stoichiometric amounts of $\mathrm{HCl}$ with the $\mathrm{Bi} / \mathrm{Cl}$ molar ratio of 1 was added into the above solution, and the resulting mixture was adjusted $\mathrm{pH}$ value to 3 with $\mathrm{Na}\left(\mathrm{CO}_{3}\right)_{2}$. After stirring for $30 \mathrm{~min}$ at room temperature, the suspension was transferred into a $50 \mathrm{~mL}$ Teflon-lined stainless steel autoclave. The autoclave was allowed to be heated at $160{ }^{\circ} \mathrm{C}$ for $6 \mathrm{~h}$ and then cooled to room temperature. The resulting precipitates were filtered and washed with ethanol and deionized water thoroughly and dried at $100{ }^{\circ} \mathrm{C}$ for $12 \mathrm{~h}$.

\subsection{Characterization}

$\mathrm{X}$-ray diffraction (XRD) measurement was employed on a XRD-6000 diffractometer using $\mathrm{Cu} \mathrm{K} \alpha$ radiation $\left(\lambda=1.5406 \AA\right.$ ) irradiated with a scanning rate of $2 \mathrm{~min}^{-1}$. Scanning electron microscopy (SEM) measurement was conducted with a JSM-6700 LV electron microscope operating at $5.0 \mathrm{kV}$. X-ray photoelectron spectroscopy (XPS) data were recorded using a Perkin-Elmer PHI 5600

\footnotetext{
${ }^{*}$ Corresponding author. Tel.: +86-024-56861711; fax: +86-02456861711. E-mail address: luguang20121101@126.com (G. Lu)
} 
electron spectrometer using achromatic $\mathrm{Al} \mathrm{K} \alpha$ radiation $(1486.6 \mathrm{eV})$ with Ar sputtering to remove the surface layer of the sample. UV-vis diffuse reflectance spectra (DRS) were recorded at room temperature with the JASCO 570 spectrophotometer equipped with an integrated sphere.

\subsection{Photocatalytic reaction}

The photocatalytic property of $\mathrm{BiOCl}$ was evaluated by decomposing rhodamine $\mathrm{B}(\mathrm{RhB})$. The reaction suspensions were obtained by adding $10 \mathrm{mg} \mathrm{BiOCl}$ powders into $200 \mathrm{~mL}$ of $10 \mathrm{mg} / \mathrm{L} \mathrm{RhB}$ aqueous solution at room temperature under vigorously stirring. The photocatalytic reaction was irritated using a $250 \mathrm{~W}$ xenon lampe equipped with a $420 \mathrm{~nm}$ cutoff filter, which can thoroughly eliminate radiation below $420 \mathrm{~nm}$ and guarantee the entire catalysis process exposed to visible light. Supernatant of RhB was obtained and the concentration of $\mathrm{RhB}$ was determined by $\mathrm{UV}$-vis spectroscopy at its characteristic wavelength of $553 \mathrm{~nm}$.

\section{RESULTS AND DISCUSSION}

Fig. 1 shows the XRD pattern of the as-synthesized $\mathrm{BiOCl}$ sample. As shown in Fig. 1, it can be seen that $\mathrm{BiOCl}$ sample shows very strong reflection peaks at $2 \theta=12.11$, $24.25,25.97,32.75,33.62,36.65,41.12$, 46.88, 49.91, $54.24,55.24,58.85,60.88$ and $68.37^{\circ}$.

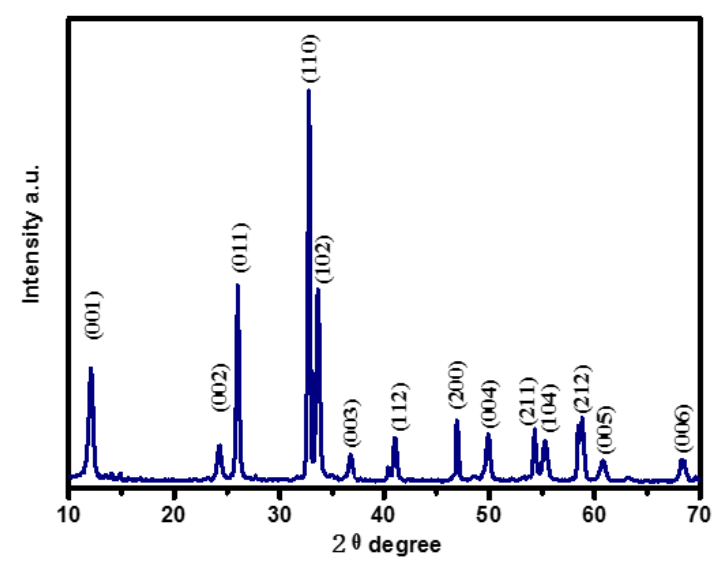

Fig. 1. Typical XRD pattern of the prepared $\mathrm{BiOCl}$ core-shell microspheres

These reflection peaks correspond to the $\mathrm{BiOCl}$ planes of (001), (002), (011), (110), (102), (003), (112), (200), (004), (221), (104), (212), (005) and (006), respectively, which can be readily indexed to the tetragonal phase of
BiOCl (JCPDS Card No. 85-0861).

No peaks of metal $\mathrm{Bi}$ or any other phases are detected, indicating that the product is very high-purity and singlephase sample. In addition, the intense and sharp diffraction peaks suggest that the as-synthesized products are wellcrystallized.

Fig. 2 shows the SEM and EDX spectrum of the asprepared $\mathrm{BiOCl}$ sample. As shown in Fig. 2 a, it is found that the fabricated $\mathrm{BiOCl}$ is microshpere with diameter about $1 \mu \mathrm{m}$. As shown in Fig. $2 \mathrm{~b}$, it is found the obtained microspheres is core-shell structure and the core or shell of these microspheres are composed of longitudinal grown nanoplates, which is different from the reported papers [12-14]. The results from EDX spectrum (Fig. 2 c) show that the as-prepared microspheres contain $\mathrm{Bi}, \mathrm{O}$ and $\mathrm{Cl}$, and no contamination elements are detected.

The chemical composition of $\mathrm{BiOCl}$ microspheres is further investigated by XPS. The XPS spectrum in Fig. 3 a reveals that as-prepared sample is composed of elements of $\mathrm{Bi}, \mathrm{O}$ and $\mathrm{Cl}$, which is in agreement with the results of EDX. As shown in Fig. 3 b, it could be seen that $\mathrm{Bi} 4 \mathrm{f}$ peaks are found at $158.8 \mathrm{eV}(\mathrm{Bi} 4 \mathrm{f} 7 / 2$ ) and $164.2 \mathrm{eV}$ (Bi 4f 5/2), indicative of $\mathrm{Bi}^{3+}$ in the obtained $\mathrm{BiOCl}$ microspheres [15].

Fig. $3 \mathrm{c}$ shows that the peak binding energy of $532.1 \mathrm{eV}$ is assigned to $\mathrm{O} 1 \mathrm{~s}$, which is arisen from oxygen in $\mathrm{BiOCl}$. Fig. $3 \mathrm{~d}$ shows the $\mathrm{Cl} 2 \mathrm{p}$ peak is associated with binding energy of $197.9 \mathrm{eV}$, which is characteristic of $\mathrm{Cl}^{-}$in $\mathrm{BiOCl}$ materials [16]. These results show that the chemical states of element composed the sample are $\mathrm{Bi}^{3+}, \mathrm{O}^{2-}$ and $\mathrm{Cl}^{-}$.

The optical properties of as-prepared $\mathrm{BiOCl}$ sample was studied using UV-vis diffuse reflectance spectra (DRS), as shown in Fig. 4 a. It is found that the $\mathrm{BiOCl}$ sample exhibits strong photo absorption in the UV region and relatively weak absorption in the visible light region. The UV-vis absorption edge of as-prepared $\mathrm{BiOCl}$ catalyst is at about $424.6 \mathrm{~nm}$.

As an indirect transition semiconductor, the band edge of $\mathrm{BiOCl}$ sample is obtained according to the energy dependence relation of:

$\alpha h v=k(h v-E g)^{1 / 2}$,

where $\alpha, h v, k$ and $E \mathrm{~g}$ are the absorption coefficient, discrete photon energy, proportionality constant and band gap energy, respectively. The plot of the $(\alpha h v)^{1 / 2}$ versus photo energy $(h v)$ of $\mathrm{BiOCl}$ sample can be fitted into a line (as shown in Fig. 4 b). The band gap energy of the $\mathrm{BiOCl}$ sample is calculated to be about $2.92 \mathrm{eV}$, implying the $\mathrm{BiOCl}$ microspheres may have visible light photoactivity.

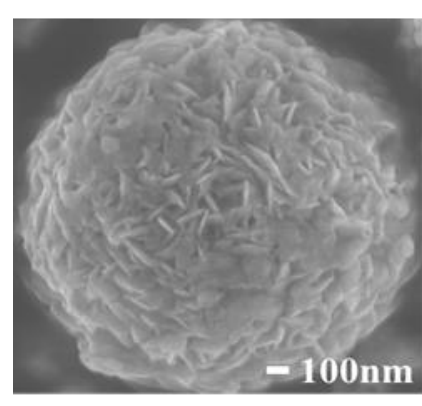

a

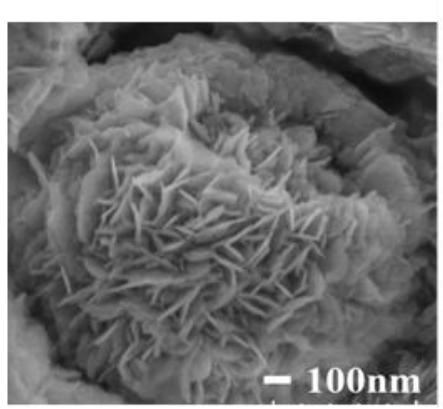

b

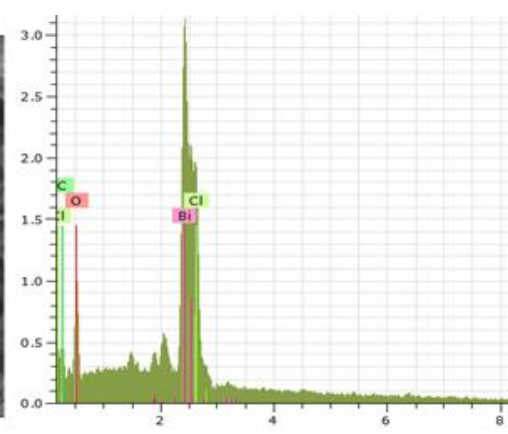

Fig. 2. a, b-SEM image; $\mathrm{c}-\mathrm{EDX}$ spectrum of the $\mathrm{BiOCl}$ core-shell microspheres 


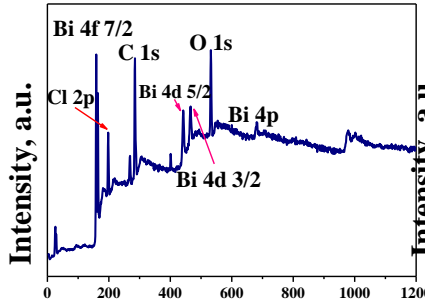

Binding energy, eV

a

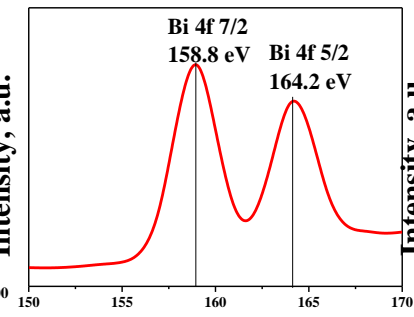

Binding energy, eV $\mathrm{b}$

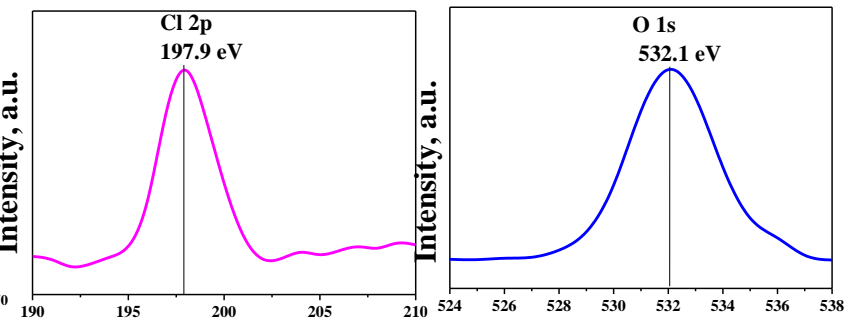

Binding energy, eV

$\mathrm{c}$
Binding energy, eV

d

Fig. 3. XPS spectra of the BiOCl core-shell microspheres: a-survey spectrum; b-Bi 4f; c-O1s; $d-C l 2 p$

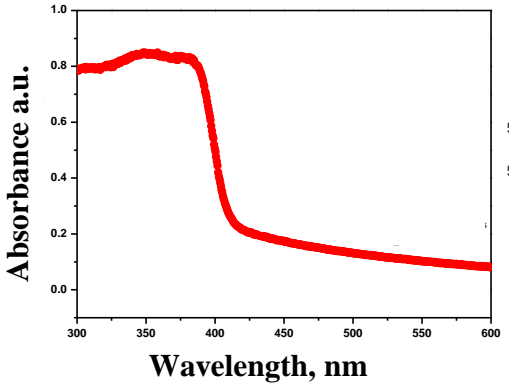

a

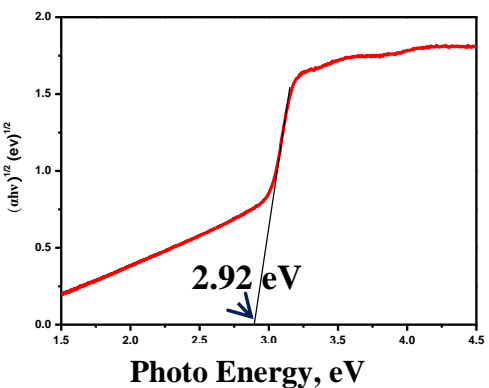

b

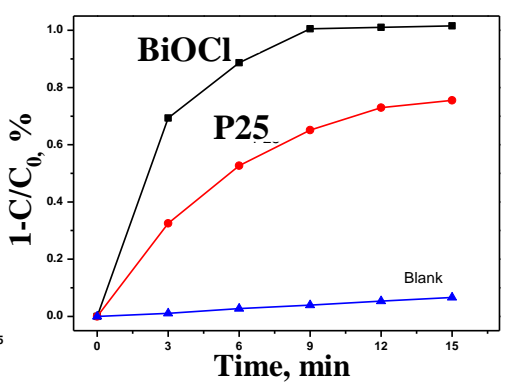

$\mathrm{c}$

Fig. 4. a-DRS spectrum; b-the $(\alpha h v)^{1 / 2}$ vs. $h v$ (b) of as-prepared BiOCl samples; c-photodegradation of RhB under visible light

Fig. $4 \mathrm{c}$ shows the photocatalytic activities of the $\mathrm{BiOCl}$ core-shell microspheres and commercial $\mathrm{TiO}_{2}(\mathrm{P} 25$, Degussa) as a comparison. In the absence of catalysts, RhB could be degraded under simulated solar light irradiation, but the reaction was very slow and only $10 \%$ of $\mathrm{RhB}$ was removed in $15 \mathrm{~min}$ irradiation. The removal efficiency was enhanced significantly in the presence of P25 or as-prepared $\mathrm{BiOCl}$ catalysts. There was $100 \%$ degradation of $\mathrm{RhB}$ for as-prepared $\mathrm{BiOCl}$ nanoplates in only $10 \mathrm{~min}$ irradiation. However, only $68 \%$ RhB molecules were decomposed using the P25 nanomaterials as photocatalyst, when the photodegradation reaction was proceeded past $15 \mathrm{~min}$. Based on these results, it can be seen that the as-prepared $\mathrm{BiOCl}$ showed about two times RhB degradation than P25.

\section{CONCLUSIONS}

The $\mathrm{BiOCl}$ samples have been prepared using solvothermal method. They are phase-pure and hierarchical microspheres with core-shell structure. The chemical states of element composed the sample are $\mathrm{Bi}^{3+}, \mathrm{O}^{2-}$ and $\mathrm{Cl}^{-}$. Moreover, the bandgap energy of the $\mathrm{BiOCl}$ product is about $2.92 \mathrm{eV}$, which shows an obvious absorption capability in the UV-vis region. The prepared $\mathrm{BiOCl}$ coreshell microspheres exhibited more excellent activity then P25 under visible light irradiation.

\section{Acknowledgments}

This work was supported by the Key Laboratory of Industrial Ecology and Environmental Engineering, China Ministry of Education (KLIEEE-13-03).

\section{REFERENCES}

$\begin{array}{llr}\text { 1. Chai, S.Y., } & \text { Kima, Y.J., } & \text { Jung, M.H., } \\ \text { Chakraborty, A.K., Jung, D., Lee, W.I. Heterojunctioned }\end{array}$
$\mathrm{BiOCl} / \mathrm{Bi}_{2} \mathrm{O}_{3}$, A New Visible Light Photocatalyst Journal of Catalysis 262 (1) 2009: pp. 144-149.

https://doi.org/10.1016/j.jcat.2008.12.020

2. Jiang, J., Zhao, K., Xiao, X.Y., Zhang, L.Z. Synthesis and Facet-Dependent Photoreactivity of BiOCl Single-Crystalline Nanosheets Journal of the American Chemical Society 134 (10) 2012: pp. $4473-4476$. https://doi.org/10.1021/ja210484t

3. Yu, C.L., Zhou, W.Q., Yu, J.C. Rapid Fabrication of BiOCI(Br) Nanosheet with High Photocatalytic Performance via Ultrasound Irradiation Chinese Journal of Inorganic Chemistry 27 2011: pp. 2033-2038.

4. Kijima, N., Matano, K., Saito, M., Oikawa, T., Konishi, T., Yasuda, H., Sato, T., Yoshimura, Y. Oxidative Catalytic Cracking of $N$-butane to Lower Alkenes over Layered BiOCl Catalyst Applied Catalysis A: General 206 (2) 2001: pp. 237-244.

https://doi.org/10.1016/S0926-860X(00)00598-6

5. Wang, W., Huang, F., Lin, X., Yang, J. Visible-LightResponsive Photocatalysts $x \mathrm{BiOBr}-(1-x) \mathrm{BiOI}$ Catalysis Communications 9 (1) 2008: pp. 8-12. https://doi.org/10.1016/j.catcom.2007.05.014

6. Henle, J., Simon, P., Frenzel, A., Scholz, S., Kaskel, S. Nanosized $\mathrm{BiOX}(\mathrm{X}=\mathrm{Cl}, \mathrm{Br}, \mathrm{I})$ Particles Synthesized in Reverse Microemulsions Chemistry of Materials 19 2007: pp. $366-373$. https://doi.org/10.1021/cm061671k

7. Feng, Y.C., Li, L., Li, J.W., Wang, J.F., Liu, L. Synthesis of Mesoporous $\mathrm{BiOBr} 3 \mathrm{D}$ Microspheres and Their Photodecomposition for Toluene Journal of Hazardous Materials 192 2011: pp. 538-544. https://doi.org/10.1016/j.jhazmat.2011.05.048

8. Deng, Z.T., Chen, D., Peng, B., Tang, F.Q. From Bulk Metal Bi to Two-Dimensional Well-Crystallized BiOX $(\mathrm{X}=\mathrm{Cl}$, $\mathrm{Br})$ Micro- and Nanostructures: Synthesis and Characterization Crystal Growth \& Design 8(8) 
2008: pp. $2995-3003$.

https://doi.org/10.1021/cg800116m

9. Ding, L.Y., Wei, R.J., Chen, H., Hu, J.C., Li, J.L. Controllable Synthesis of Highly Active BiOCl Hierarchical Microsphere Self-Assembled by Nanosheets with Tunable Thickness Applied Catalysis B: Environmental 172-173 2015: pp. $91-99$.

https://doi.org/10.1016/j.apcatb.2015.02.019

10. Hao, H.Y., Xu, Y.Y., Liu, P., Zhang, G.Y. $\mathrm{BiOCl}$ Nanostructures with Different Morphologies: Tunable Synthesis and Visible-Light-Driven Photocatalytic Properties Chinese Chemical Letters 26 2015: pp. 133-136. https://doi.org/10.1016/j.cclet.2014.11.022

11. Wu, Y.P., Zhou, Z.H., Tuo, Y.F., Huang, Y., Shen, S. A Novel and Facile Method to Synthesize Self-Assembled BiOI Microrings Composed of Nanoplatelets Materials Letters 111 2013: pp. 43-46.

https://doi.org/10.1016/j.matlet.2013.08.031

12. Cao, S.H., Guo, C.F., Lv, Y., Guo, Y.J., Liu, Q. A Novel BiOCl Film with Flowerlike Hierarchical Structures and Its Optical Properties Nanotechnology 20 2009: pp. 275702. https://doi.org/10.1088/0957-4484/20/27/275702
13. Lu, G., Wang, H., Zou, X.J. Effect of Drying Temperatures on Structural Performance and Photocatalytic Activity of BiOCl Synthesized by A Soft Chemical Method Journal of Solid State Chemistry 239 2016: pp. 259-264. https://doi.org/10.1016/j.jssc.2016.04.039

14. Sanaa, S.K.I., Vladimir, U., Yulia, K., Ella, M., Inna, P., Yoel, S. A New Family of $\mathrm{BiO}(\mathrm{ClxBr} 1-\mathrm{x})$ Visible Light Sensitive Photocatalysts Catalysis Communications 12 (12) 2011: pp. 1136-1141. https://doi.org/10.1016/j.catcom.2011.03.014

15. Song, S.Y., Gao, W., Wang, X., Li, X.Y., Liu, D.P., Xing, Y., Zhang, H.J. Microwave-Assisted Synthesis of BiOBr/Graphene Nanocomposites and Their Enhanced Photocatalytic Activity Dalton Transactions 41 2012: pp. $10472-10476$. https://doi.org/10.1039/C2DT31088K

16. Ai, Z.H., Ho, W.K., Lee, S.C. Efficient Visible Light Photocatalytic Removal of $\mathrm{NO}$ with $\mathrm{BiOBr}-\mathrm{Graphene}$ Nanocomposites Journal of Physical Chemistry C 115 2011: pp. $25330-25337$. https://doi.org/10.1021/jp206808g 